\title{
WEAK INTERACTIONS
}

\section{MEASUREMENT OF THE NEUTRON LIFETIME BY COUNTING TRAPPED PROTONS}

\author{
M.S. Dewey, D.M. Gilliam, and G.L. Greene \\ National Institute of Standards and Technology, Gaithersburg, Maryland 20899 \\ C.D. Keith and W.M. Snow \\ Indiana University, Bloomington, Indiana 47408 \\ J. Pauwels, A. Lamberty, and R. Eykens \\ Central Bureau for Nuclear Measurements, B-2440 Geel, Belgium \\ R.D. Scott \\ Scottish Universities Research and Reactor Center, East Kilbride, \\ Glasgow G75 0QU, United Kingdom
}

The decay rate of the neutron is an important parameter in low energy weak interactions. In combination with other measurements of neutron decay parameters, such as the electron asymmetry coefficient in polarized neutron decay, it determines the weak polar vector-axial vector coupling ratio $\lambda=\frac{g_{A}}{g_{V}}$. By comparison with muon decay, the KM matrix element $V_{u d}$, one of the parameters of the Standard Model of elementary particle interactions, can be determined. It is important to note that present measurements in the neutron sector are marginally inconsistent with the unitarity of the Kobayashi-Maskawa (KM) matrix: ${ }^{1-3}$ the value of $V_{u d}$ inferred from one of the two $A$ measurements of comparable accuracy causes a violation of the unitarity of the KM matrix by $3 \sigma$. Further measurements are required to resolve this discrepancy and to search for indications of physics beyond the Standard Model, such as the possible existence of right-handed weak currents.

In addition, the neutron decay rate influences theoretical predictions relating to Big Bang Nucleosynthesis (BBN) of the primordial helium abundance in the universe and the number of different types of light neutrinos $N_{\nu}$ (and therefore the number of generations) in the Standard Model. The most recent comparison of BBN helium abundance calculations to observation constrains $N_{\nu} \leq 3.04,{ }^{4}$ consistent with and complementary to the value derived from $Z_{0}$ decay, $N_{\nu}=2.985 \pm 0.012_{\text {expt }} \pm 0.019_{\text {theory }} .{ }^{5}$ However, new measurements ${ }^{6}$ of the primordial abundance ratio of deuterium to hydrogen may indirectly change the $\mathrm{BBN}$ prediction for the helium abundance by modifying the "best" value for the photon to baryon ratio $\eta$. For a given value of $\eta$, about $90 \%$ of the uncertainty in the BBN prediction for the primordial helium abundance comes from the accuracy of the neutron decay rate. ${ }^{7}$ Improved neutron decay rate measurements are therefore important for sharpening the $\mathrm{BBN}$ prediction. 
An experiment is in progress at the NIST Cold Neutron Research Facility to measure the decay rate of the neutron. ${ }^{8}$ The measurement is performed by confining the protons from in-beam neutron decays in a Penning trap and counting the trapped protons while simultaneously monitoring the neutron flux passing through the trap. The goal of this collaboration is to perform a measurement of the neutron lifetime with an accuracy at the $0.1 \%$ level. A previous version of the experiment reached an accuracy of $0.5 \% .^{9}$ The value for the neutron lifetime recommended by the Particle Data Group is $\tau_{n}=889.1 \pm 2.1$ seconds. ${ }^{10}$

The experiment has been running for several months at the NIST Cold Neutron Research Facility. Approximately eight million decay events have been recorded to date. Additional running is planned in late 1995. Data analysis is in progress. Work on the absolute calibration of the neutron flux monitor will continue both during and after the decay data has been taken.

1. A.S. Carnoy, et al., Phys. Rev. Lett. 65, 3249 (1990).

2. J.C. Hardy, et al., Nucl. Phys. A509, 429 (1990).

3. I.S. Towner, Nucl. Phys. A540, 478 (1992).

4. P.J. Kernan and L.M. Krauss, Phys. Rev. Lett. 72, 3309 (1994).

5. ALEPH collaboration, Phys. Lett. B321, 168 (1994); DELPHI collaboration, Phys. Lett. B276, 536 (1992); L3 collaboration, Z. Phys. C51,179 (1991); OPAL collaboration, Z. Phys. C61, 19 (1994).

6. A. Songaila, et al., Nature 368, 599 (1994).

7. M.S. Smith, L.H. Kawano, and R.A. Malaney, Astrophys. J. Suppl. 85, 219 (1993).

8. M.S. Dewey, et al., Proceedings of the Third International Symposium on Weak and Electromagnetic Interactions in Nuclei, ed. T.D. Vylov (World Scientific, New York, 1992), p. 44.

9. J. Byrne, et al., Phys. Rev. Lett. 65, 289 (1989).

10. Particle Data Group, Phys. Rev. D 45, S1 (1992). 